\title{
Apoios de Decisão: Instrumento de Auxílio à Medicina Baseada em Preferências. Uma Revisão Conceitual
}

\section{Decision Aids: an Instrument to Eliciting Patient Preference. Current Concepts}

\author{
Mirhelen Mendes de Abreu $^{(1)}$, Sérgio Candido Kowalski(2), Rozana M. Ciconelli( ${ }^{(3)}$, Marcos Bosi Ferraz ${ }^{(4)}$
}

\section{RESUMO}

A necessidade da incorporação das preferências dos pacientes nas tomadas de decisão é uma realidade da assistência médica contemporânea, que caracteriza a medicina baseada em preferências. Os apoios de decisão são instrumentos que surgem como mecanismos de auxílio ao médico a expor riscos e benefícios de uma determinada tomada de decisão, sendo um mecanismo facilitador para a decisão compartilhada. Na literatura médica, particularmente em reumatologia, nota-se um emergente interesse pelo estudo das preferências dos pacientes nos ensaios clínicos. Este artigo de revisão tem por objetivo conceituar e caracterizar os apoios de decisão e suas implicações na pesquisa e prática clínicas, bem como ressaltar os princípios metodológicos necessários para a sua utilização e desenvolvimento.

Palavras-chave: apoios de decisão, quadro de decisão, preferências dos pacientes, medicina baseada na valoração.

\section{INTRODUÇÃO}

Nas últimas décadas, observa-se um aumento na ênfase da participação do paciente em uma tomada de decisão médica, como alternativa ao modelo paternalista, no qual o médico decide e delibera a respeito de todos os passos do processo propedêutico e/ou terapêutico. Esse modelo de relacionamento, chamado decisão compartilhada, não pode ser confundido com o consentimento informado, em que o médico apresenta as questões, até expõe os riscos, mas, ao paciente, cabe incorporar a decisão. Muito além de expor riscos e benefícios, decisão compartilhada se caracteriza por um processo no qual ambos médicos e pacientes analisam as evidências disponíveis para uma determinada questão clínica, incluindo as opções e conseqüências, incorporando as preferências dos pacientes para os desfechos e estados de

\begin{abstract}
Patient preferences in decision making is one reality of the medical assistance, which characterizes the value based medicine. The decision aids are instruments that appear as support mechanisms for the doctor to exercise this model of decision making. In medical literature, particularly in rheumatology, an emergent interest on patient's preference study is noticed in clinical trials. The aim of this article is to appraise and to characterize the decision aids and its implications in the practical research and clinics, as well as standing out the necessary methodology principles for its use and development.
\end{abstract}

Keywords: decision aids, decision board, patient preference, value-medicine enbased.

saúde que advêm de cada alternativa ${ }^{(1,2)}$.

Algumas associações médicas têm procurado recomendar decisão compartilhada em suas diretrizes, principalmente em questões de promoção de saúde, refletindo, em parte, o reconhecimento de que determinadas questões preventivas envolvem trocas complexas (trade-offs) entre riscos e benefícios. A Sociedade Americana de Câncer (American Cancer Society - ACS), Associação Americana de Urologia (American Urological Association-AUC) e o Colégio Americano de Medicina (American College of Physicians - ACP) recomendam a tomada de decisão compartilhada em suas diretrizes para o rastreio de câncer de próstata ${ }^{(3,4)}$. Semelhantemente, a National Institutes of Health (NIH) recomenda, em seus consensos, a tomada de decisão compartilhada em suas diretrizes para câncer de próstata e para a realização de mamografia para mulheres entre 40-49 anos de idade ${ }^{(5,6)}$.

\footnotetext{
Recebido em 30/03/06. Aprovado, após revisão, em 15/07/06.

1. Pós-graduanda da Disciplina de Reumatologia da Escola Paulista de Medicina da Universidade Federal de São Paulo (UNIFESP).

2. Médico do Serviço de Reumatologia do Hospital das Clínicas da Faculdade de Medicina da Universidade de São Paulo (USP).

3. Médica Assistente Doutora da Disciplina de Reumatologia da UNIFESP, Membro do Projeto Pronuclear da Sociedade Brasileira de Reumatologia (SBR).

4. Professor Adjunto da Disciplina de Reumatologia da UNIFESP.

Endereço para correspondência: Mirhelen Mendes de Abreu, Rua Botucatu, 740, $3^{\circ}$ andar, Ed. Jairo Ramos, São Paulo, SP, Brasil, telefone/fax: (11) 5579-6665,

e-mail:mirhelen@terra.com.br
} 
Na prática clínica, freqüentemente nos deparamos com situações de incertezas científicas quanto às conseqüências (em perfis de risco e benefício) de uma tomada de decisão(7). Neste cenário, incorporar as preferências do paciente (que é quem terá de conviver com as conseqüências da decisão efetuada) torna-se um exercício ético e democrático do cotidiano médico ${ }^{(8)}$.

No mundo contemporâneo, as informações científicas expostas em sites da internet permitem que o paciente conheça diferentes estratégias para se conduzir uma dada questão clínica. É de se notar, contudo, que tais informações nem sempre são baseadas em dados científicos e, em muitas situações, acabam dificultando o paciente ao processar seus valores e preferências, principalmente, por apresentar, em muitas situações, um conteúdo sensacionalista e/ou com expectativas irreais ${ }^{(9)}$. Transmitir esses conhecimentos ao público leigo, de modo a expor, de forma clara e racional, as incertezas dos desfechos e os riscos de cada alternativa tem se tornado foco de pesquisas científicas em diferentes níveis de promoção de saúde ${ }^{(10)}$. Cabe ressaltar, no entanto, que o reconhecimento da necessidade de ter acesso a informações pertinentes à sua doença, particularmente no contexto das doenças crônicas, implica em admitir o paciente como sujeito ativo no processo de relacionamento médico-paciente, cujas necessidades e valores devem ser incorporados em uma tomada de decisão ${ }^{(11)}$.

Os apoios de decisão (suporte de decisão compartilhada) podem ser definidos como ferramentas de auxílio ao médico a expor diferentes estratégias e suas respectivas conseqüências em riscos e benefícios para uma dada questão clínica ${ }^{(12,13)}$. Trata-se de uma estratégia que tem como fundamento básico facilitar ao paciente analisar as alternativas propostas e processar seus valores e preferências para cada possível risco e benefício, respeitando-se os preceitos metodológicos para a sua utilização ${ }^{(14)}$.

A ciência médica tem se ampliado vertiginosamente no sentido de desenvolver apoios de decisão como ferramentas sólidas e capazes de atingir tal fundamento. Neste sentido, o presente texto visa conceituar os princípios e características de um apoio de decisão, bem como salientar sua participação nas pesquisas científicas e na prática clínica.

\section{PRINCÍPIOS BÁSICOS}

A característica principal de um apoio de decisão é conter mais de uma estratégia, com riscos e benefícios distintos para a mesma questão clínica. Todos os aspectos que envolvem este instrumento devem se norteados por esta diretriz. Especialistas nesses instrumentos da Cochrane
Collaboration definiram critérios de padrão de qualidade de um apoio de decisão, conhecidos como CREDIBLE (Component developers and development; Recent; Evidence based; Devoid of conflicts of Interest; Balanced presentation of options, benefits, harms; Efficacious). Em outras palavras, todo apoio de decisão deve apresentar um conteúdo atualizado, baseado em níveis de evidência disponíveis, deve ser livre de conflito de interesse, possuir um conteúdo real e equilibrado quanto as chances dos eventos (riscos e benefícios) e ser eficaz na prática clínica ${ }^{(15)}$.

Para que tais princípios sejam respeitados, faz-se necessário uma revisão na educação médica, visto que este modelo reformula o relacionamento médico-paciente ${ }^{(16)}$. A relação de poder é compartilhada, bem como a relação com as responsabilidades. Neste paradigma, este mecanismo pode, hipoteticamente, otimizar a adesão do paciente ao tratamento, o que traria conseqüências benéficas no maior alcance dos desfechos clínicos almejados. Em resumo, a utilização de um apoio de decisão é um exercício da medicina baseada nas preferências ${ }^{(17,18)}$.

PERFIS DE RISCOS E BENEFÍCIOS DISTINTOS: TOMADA DE DECISÃO EM MEIO ÀS INCERTEZAS

Antes de prosseguirmos com esta seção, cabe revisitar as definições de certeza, incerteza e risco a fim de nortear o raciocínio deste tópico e analisar suas relações com o cotidiano médico de tomada de decisão. Em conformidade com a teoria da utilidade, temos que as tomadas de decisão ocorrem nos seguintes contextos:

Sob certeza: quando uma ação acarreta em um determinado ganho (com 100\% de chance);

Sob incerteza: quando todas as ações possíveis levam a um conjunto de ganhos possíveis mas as probabilidades associadas são desconhecidas (e não $100 \%$ );

Sob risco: quando existe uma probabilidade ou possibilidade da ocorrência de valores para determinados eventos e fenômenos, sejam eles benéficos, indesejáveis e/ou adversos.

Assim, processos como medições, observações, avaliações e tomadas de decisão podem ser influenciados por várias fontes de incertezas. Isto leva a estabelecer a convivência contínua e inevitável com inúmeros tipos de risco ${ }^{(19)}$.

A comunicação baseada em riscos é uma característica da medicina contemporânea ${ }^{(20,21)}$. No entanto, nem sempre conhecemos os riscos das decisões a serem feitas. Wennberg et $a^{(22)}$ definiram tomada de decisão em duas grandes classes, as quais chamaram de decisão efetiva e decisão centrada nas preferências. Esta classificação se baseia na força do 
nível de evidência científica disponível e na magnitude das relações de risco e benefício. Sendo assim, temos que:

a) decisão efetiva: quando as reais chances de eventos são claros e bem definidos. O benefício é comprovado e nitidamente superior ao prejuízo. Exemplo: o uso de bisfosfonatos para o tratamento da osteoporose e suas implicações na prevenção de fratura óssea.

b) decisão centrada nas preferências: quando o perfil de risco-benefício apresenta um limiar estreito ou quando tais conseqüências não são definidas. Exemplo: terapia de reposição hormonal em mulheres no climatério. Neste exemplo, a nítida resposta aos sintomas do climatério, bem como o impacto positivo na prevenção de fraturas ósseas devem ser contrabalanceados pelo seu potencial em desenvolver câncer de mama e risco de doença cardiovascular. Esta tomada de decisão deve ser compartilhada com a paciente. Deve-se notar que, uma vez que o médico não cumpre com o seu papel de orientar e auxiliar ao paciente,

TABELA 1

CARACTERÍSTICAS DOS TIPOS BÁSICOS DE DECISÃO E O USO APROPRIADO DOS APOIOS DE DECISÃO ${ }^{(22)}$

\begin{tabular}{|c|c|}
\hline $\begin{array}{c}\text { EFETIVIDADE } \\
\text { (Benefícios superam malefícios) }\end{array}$ & $\begin{array}{c}\text { PREFERÊNCIA } \\
\text { (Relação risco/benefício incerto) }\end{array}$ \\
\hline \multicolumn{2}{|c|}{ Clareza da decisão } \\
\hline Recomendação padronizada & Não tem padrão estabelecido \\
\hline Força de evidência disponível & Força de evidência disponível \\
\hline $\begin{array}{l}\text { Papel do paciente: conhecer a } \\
\text { realidade }\end{array}$ & $\begin{array}{l}\text { Papel do paciente: analisar riscos, } \\
\text { benefícios, incertezas }\end{array}$ \\
\hline \multicolumn{2}{|c|}{ Clareza dos valores } \\
\hline $\begin{array}{l}0 \text { potencial benefício supera a } \\
\text { chance de prejuízo? }\end{array}$ & $\begin{array}{l}\text { Opção única: o potencial benefício } \\
\text { é mais importante que a incerteza } \\
\text { do risco? } \\
\text { Múltiplas decisões: os potenciais } \\
\text { benefícios são mais importantes que } \\
\text { as chances de riscos? }\end{array}$ \\
\hline \multicolumn{2}{|c|}{ Características dos apoios de decisão } \\
\hline $\begin{array}{l}\text { Estágios e barreiras para as } \\
\text { mudanças }\end{array}$ & $\begin{array}{l}\text { Estágios e barreiras para a tomada } \\
\text { de decisão }\end{array}$ \\
\hline $\begin{array}{l}\text { Ferramentas para as motivações } \\
\text { e barreiras: informações; } \\
\text { motivação }\end{array}$ & $\begin{array}{l}\text { Ferramentas para analisar incertezas: } \\
\text { informações, } \\
\text { clareza dos valores. }\end{array}$ \\
\hline $\begin{array}{l}\text { Facilitação do aprendizado para } \\
\text { direções futuras }\end{array}$ & $\begin{array}{l}\text { Facilitação do processo de } \\
\text { aprendizado para direções futuras }\end{array}$ \\
\hline \multicolumn{2}{|c|}{ Avaliação } \\
\hline $\begin{array}{l}\text { Processo: conhecimento, } \\
\text { conflito decisional, progresso } \\
\text { em estágio de mudança }\end{array}$ & $\begin{array}{l}\text { Processo: conhecimento, percepção } \\
\text { realística dos riscos, conflito } \\
\text { decisional, progresso no estágio da } \\
\text { tomada de decisão }\end{array}$ \\
\hline $\begin{array}{l}\text { Maior comprometimento com o } \\
\text { tratamento? (Adesão) }\end{array}$ & $\begin{array}{l}\text { Maior comprometimento com o } \\
\text { tratamento? (Adesão) }\end{array}$ \\
\hline
\end{tabular}

ele irá buscar em outras fontes que, na maioria das vezes, não são construtivas nem para a sua saúde nem para a relação com o seu médico (Tabela 1 ), além de acarretar, freqüentemente, na solicitação de métodos propedêuticos dispendiosos e, nem sempre, necessários ${ }^{(22)}$. A Figura 1 ilustra os principais contextos de certeza/incerteza e risco e suas aplicações nas tomadas de decisão médica ${ }^{(23)}$.

Figura 1

MODELOS DE DECISÃO MÉDICA EM DIFERENTES CONTEXTOS DE INCERTEZA (ADAPTADO) $)^{(23)}$

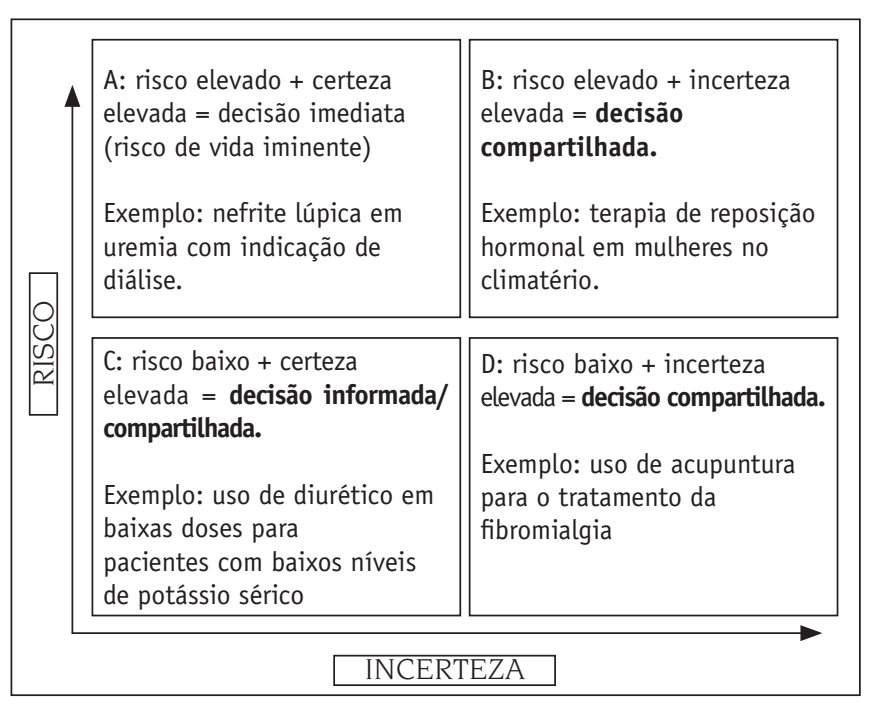

Baseado nesses conceitos, Giffiths et a ${ }^{(24)}$ desenvolveram um estudo qualitativo que procurou descrever como os médicos lidam com as incertezas inerentes às evidências científicas em suas consultas médicas. O cenário do trabalho envolveu a questão da terapia de reposição hormonal, densitometria óssea e rastreio do câncer de mama. Participaram do estudo mulheres de 45-64 anos e foram avaliadas 109 consultas médicas ( 73 de médicos generalistas e 36 de especialistas). As consultas foram categorizadas em três níveis de certeza: 1) foco na certeza evidente do benefício; 2) coerente acúmulo de evidência dos riscos e benefícios, mas com as incertezas não explícitas pelos principais trabalhos e 3) conhecimento da incerteza em nível de evidência científica quanto à questão proposta. Em sua análise, o autor apontou que, em geral, quando a situação é de incerteza, o médico procura tomar decisões provisórias e solicitar exames, criando uma situação de certeza mítica e irreal. A importância deste estudo está em apontar a necessidade de uma reformulação na mentalidade do médico em expor o desconhecimento para um determinado cenário ${ }^{(24)}$. 
OS APOIOS DE DECISÃO NA PESQUISA CIENTÍFICA: PERGUNTAS, OBJETIVOS E MEDIDAS DE DESFECHO A SEREM AVALIADAS

Aliado ao vertiginoso interesse científico pelo desenvolvimento adequado dos apoios de decisão, surgem algumas discussões envolvendo as propriedades destes instrumentos. As questões e controvérsias mais freqüentes na literatura são: (1) quanto à relevância pertinente do conteúdo a ser exposto; (2) quanto ao modelo ideal do apoio de decisão; (3) quanto aos objetivos a serem alcançados nos ensaios clínicos que visem estudar estes instrumentos e, por último, (4) quanto às medidas de desfecho a serem avaliadas nos ensaios clínicos ${ }^{(25)}$.

O principal foco de debate dos apoios de decisão envolvem a relevância do conteúdo a ser exposto ${ }^{(26,27)}$. Muitos autores consideram que tal relevância está diretamente relacionada ao contexto da questão clínica ${ }^{(28)}$. Assim, se o foco da discussão for, por exemplo, a indicação de uma biópsia renal para a condução do tratamento de uma nefrite lúpica, o apoio de decisão deve conter as vantagens, em números, de se realizar o procedimento, os riscos de complicação do procedimento, bem como as conseqüências reais em não fazê-lo. A decisão final deve ser do paciente, o que potencializa a responsabilidade do médico ${ }^{(29)}$. Decisões menos complexas, como o tipo de droga modificadora de atividade de doença (DMARDs) mais apropriado para o tratamento da artrite reumatóide (AR) deve envolver os benefícios do tratamento, as conseqüências do não-tratamento, bem como os efeitos adversos de cada opção. É fundamental comentar que efeitos adversos tais como náuseas, vômitos e queda de cabelo são questões reais que devem ser discutidas com o paciente (independentemente do seu nível sociocultural), visto que são problemas do seu dia-a-dia que vão contribuir para sua adesão ao tratamento a longo prazo ${ }^{(30)}$.

Quanto ao modelo (formato) ideal do apoio de de-

Figura 2:

ÁrVore DE DECISÃo HIPOTÉTICA (SCHWARTZ ET AL. 1973). ADAPTADO (REF.32)

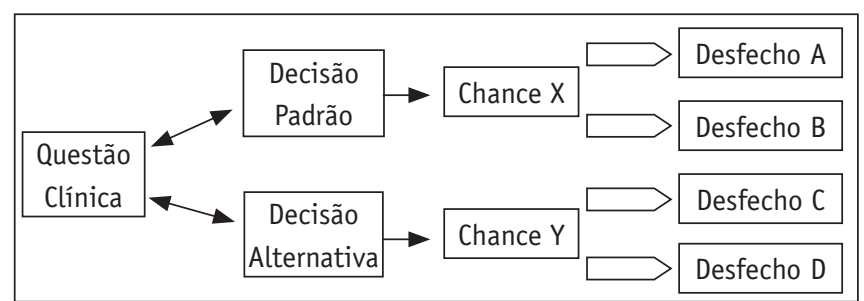

Esqueleto básico de uma árvore de decisão. cisão, a principal discussão é qual o melhor formato está relacionado a que tipo de doente/questão clínica. Gafni et $a^{(31)}$ descrevem que o melhor formato deve acompanhar a arquitetura de uma árvore de análise decisão, onde as chances e as conseqüências vão sendo expostas progressivamente, direcionadas pelas escolhas do paciente. Neste modelo, o formato que melhor se aplica é o quadro de decisão ${ }^{(31)}$. A Figura 2 ilustra um modelo esquemático de árvore de análise de decisão ${ }^{(32)}$.

Por outro lado, O'Connor et $a^{(33)}$ conduziram uma revisão sistemática em que foram analisadas as características dos apoios de decisão. Dentre outros aspectos, o estudo mostrou que formatos mais detalhados, com recursos de informática, têm um impacto maior na melhora do conhecimento do paciente bem como na redução do seu conflito decisional, sendo apontados, pelos autor, como o modelo mais apropriado. No entanto, esta observação não teve significância estatística no estudo desenvolvido ${ }^{(33)}$.

O terceiro aspecto diz respeito aos objetivos a serem alcançados a partir dos ensaios clínicos que utilizem esta ferramenta. Por princípio de rigor metodológico, tais objetivos devem ser o alicerce através do qual as medidas de desfecho serão mensuradas. Neste sentido, o(s) objetivo(s) deve(m) ser consistente (s) com o sentido de escolha, porque esta é a essência do apoio de decisão. Os objetivos genéricos visam promover ao paciente conhecimentos relevantes quanto aos riscos e benefícios para uma tomada de decisão consciente e auxiliar aos pacientes a terem clareza dos seus valores. Os objetivos específicos devem estar em consonância com os aspectos clínicos a serem valorados ${ }^{(34)}$.

Quanto aos desfechos a serem mensurados nas pesquisas científicas, os mais relevantes incluem: (a) o conhecimento do paciente; (b) a satisfação com o processo de tomada de decisão; (c) o impacto psíquico da decisão (d) o impacto no status de saúde (Health related quality of life) e (e) o impacto econômico ${ }^{(35)}$.

O conhecimento do paciente pode ser analisado por escalas de conhecimento ou questionários específicos para o paciente. Aqui, as discussões residem quanto ao caráter do conhecimento necessário (se subjetivo, baseado no quanto o paciente refere ter compreendido ou objetivo, a partir de perguntas específicas sobre o conteúdo exposto). Outra discussão é se o conhecimento a ser medido deve estar relacionado com os aspectos clínicos da doença ou com a noção de risco que cada alternativa expõe $e^{(36)}$.

A satisfação com o processo de decisão é um aspecto no qual O'Connor et $a^{\left({ }^{37)}\right.}$ apontam como sendo uma das principais contribuições dos apoios de decisão. Segundo os 
resultados de suas observações, a satisfação com o processo de decisão e com a decisão propriamente dita reduzem o conflito decisional e a ansiedade do paciente, além de otimizar a qualidade do tempo de consulta médica ${ }^{(37)}$.

Ainda não temos disponível estudos que apontem para o impacto econômico dos apoios de decisão. Os estudos que se propuserem a alcançar tais objetivos devem considerar se as escolhas feitas de modo mais consciente orientam por condutas mais conservadoras (e, portanto, de menor custo) ou não. Cumpre ressaltar que, por princípio, não existe escolha certa ou errada e se, a partir de um apoio de decisão, a tendência for por medidas mais invasivas (com suas respectivas implicações econômicas), esta deverá ser respeitada. Parece, no entanto, que a tendência de um paciente mais consciente é de escolher por condutas mais conservadoras, mas esse aspecto ainda não está claro $^{(38)}$. Outro ponto a ser considerado é quanto ao custo da implementação dessas ferramentas. Ainda não dispomos de dados concretos mas, ao que parece, o quadro de decisão é um meio mais barato a ser utilizado, visto que dispõe de material ilustrativo e não requer conhecimentos em informática, como ocorre com os demais formatos. Considerando a escassez dos recursos, principalmente nos países subdesenvolvidos, talvez este seja o modelo ideal a ser implantado na prática clínica ${ }^{(39)}$.

Os conceitos expostos nos tópicos anteriores podem ser traduzidos à beira do leito à partir da comunicação do médico com o seu doente baseada em riscos e benefícios, de modo claro, conciso e considerando as reais probabilidades de ocorrerem os desfechos, utilizando uma linguagem apropriada ao seu nível cultural e sua experiência pessoal.

Para comprovar sua utilidade na melhoria da asssitência médica, Kawamoto et al ${ }^{(40)}$ conduziram uma revisão sistemática com o objetivo de tentar identificar as características dos apoios de decisão que contribuem com a melhora da assistência médica. Nos critérios de seleção, incluiu estudos que avaliaram a capacidade de um apoio de decisão em melhorar a prática clínica. Os estudos foram analisados quanto às significâncias clínica e estatística em melhorar a assistência médica a partir de 15 características mais citadas na literatura. Tais características podem ser subdivididas em quatro categorias, a saber: características genéricas; interação do médico com a ferramenta de apoio; características do conteúdo exposto e características auxiliares. Os autores analisaram 70 estudos, dos quais, $68 \%$ mostraram melhora na prática clínica com o uso dos apoios de decisão. Os critérios definidos para se julgar uma melhora na assistência médica foram: melhora no conhecimento da doença; redução do conflito decisional; participação ativa do paciente na tomada de decisão (decisão médica compartilhada) e, por último, satisfação com o processo de tomada de decisão e com a decisão propriamente dita. A análise de regressão logística revelou que quatro das características apontadas foram preditores isolados de melhora na prática clínica, a saber: incorporação dos apoios de decisão como uma ferramenta de trabalho do médico $(\mathrm{p}<0,00001)$; conteúdo real quanto as chances de ocorrerem os desfechos analisados ( $\mathrm{p}=0,0187)$; presença do apoio de decisão no tempo e local da tomada de decisão $(\mathrm{p}=0,0263)$ e utilização de recursos de computador ( $\mathrm{p}=0,0294)$. Dos 32 estudos cujos apoios apresentaram estas características, 30 (94\%) apresentaram significante melhora na prática clínica. Em conclusão, os autores apontam para a importância da implementação dos apoios de decisão na prática clínica e sugerem que, quando factíveis, tais ferramentas contenham tais características ${ }^{(40)}$.

\section{PERSPECTIVAS FUTURAS}

A análise de decisão centrada no paciente é um conceito amplo, que se caracteriza por avaliar a melhor escolha para um paciente em particular, envolvendo um cenário clínico específico, em que ganhos e perdas em saúde devem ser analisados de modo concreto, considerando os reais valores dos pacientes $^{(41)}$.

O futuro da ciência da decisão engloba o desenvolvimento dos suportes de decisão, devendo-se estimar seu impacto potencial e real para a assistência médica. Existem muitos hiatos nas pesquisas, dentre os quais citamos: (1) persistência na escolha tomada; (2) efeitos na interação médico-paciente; (3) o impacto econômico, dentre outros. Os profissionais que desenvolvem e usam os apoios de decisão necessitam de um consenso com um mínimo de critério para avaliar seus efeitos à curto e médio pra$\mathrm{zo}^{(42)}$. Outro desafio incipiente envolve a incorporação desses princípios de preferências individuais nas decisões coletivas, particularmente, para a realidade das doenças reumáticas crônicas. Cumpre ressaltar que têm emergido estudos que procuram avaliar as implicações dos apoios de decisão em pacientes com baixo nível de escolaridade, bem como o instrumento mais apropriado para esse público em questão ${ }^{(43,44)}$.

Declaramos a inexistência de conflitos de interesse. 


\section{REFERÊNCIAS}

1. Charles C, Gafni A, Whelan T: Shared decision making in the medical encounter: what does it mean? (or it takes at least two to tango). Soc Sci Med 44: 681-92, 1997.

2. Brody DS: The patient's role in clinical decision making. Ann Intern Med 93: 718-22, 1980.

3. von Eschenbach A, Ho R, Murphy GP, Cunningham M, Lins $\mathrm{N}$ : American Cancer Society guideline for the early detection of prostate cancer: update. CA Cancer I Clin 47: 261-4, 1997.

4. Middleton RG, Thompson IM, Austenfeld MS et al: Prostate Cancer Clinical Guideline Panel: Report on the Management of Clinically Localized Prostate Cancer. Baltimore. MD: American Urological Association, 1995.

5. Coley CM, Barry MJ, Mulley AG: Early detection of prostate cancer. Clinical Guideline: Part III. Ann Intern Med 126: 4804, 1997.

6. National Institute of Health Consensus Development Panel. National Institute of Health consensus development conference statement : Breast cancer screening for women ages 40-49. J Natl Cancer Inst 89: 1015-26, 1997.

7. Graham JD, Wiener JB: Risk versus risk: tradeoffs in protecting health and the environment. NEJM 335: 140-41, 1996.

8. Asadi-Lari M, Tamburini M, Gray D: Patients' needs, satisfaction, and health related quality of life: towards a comprehensive model. Health Qual Life Outcomes 2:32, 2004.

9. Turney J: Public understanding of science. Lancet 347: 1087-90, 1996.

10. Woolf SH, Chan ECY, Harris R, Sheridan SL et al: Promoting informed choice: transforming health care to dispense knowledge for decision making. Ann Intern Med 143: 293-300, 2005.

11. McNutt RA: Shared medical decision making: problems, process, progress. JAMA 292: 2516-18, 2004.

12. Whelan T, Levine M, Gafni A, et al : Mastectomy or lumpectomy? Helping women make informed choices. J Clin Oncol 17: 1727$35,1999$.

13. Barry MJ: Health decision aids to facilitate shared decision making in office practice. Ann Intern Med 136: 127-35, 2002.

14. O'Connor AM, Rostom A, Fiset V et al: Decision aids for patients facing health treatment or screening decisions: systematic review. BMJ 319: 731-34, 1999.

15. Cochrane Inventory of Patient Decision Aids. Otawa: Otawa Health Research Institute. Acesso em http://2004.187.39.28/ cochinvent.php, em 12 de abril de 2005.

16. Thomasma DC: Beyond medical paternalism and patient autonomy: a model of physician conscience for the physicianpatient relationship. Ann Intern Med 98: 243-8, 2002.

17. Sheridan SL, Harris RP, Woolf SH: Shared decision-making workgroup of the U.S. Preventive Services Task Force. American J Preventive Med 26: 56-66, 2004.

18. Degner LF, Kristjanson LJ, Bowman D, et al: The patient-physician relationship: Information needs and decisional preferences in women with breast cancer. JAMA 277: 1485-92, 1997.

19. Petitti DB. In: Meta-analysis, decision analysis and costeffectiveness analysis. Methods for quantitative synthesis in Medicine. $2^{\underline{a}}$ Ed. Oxford, Oxford University Press, 2000.

20. Woloshin S, Schwartz LM, Moncur M, Gabriel S, Tosteson
AN: Assessing values for health: numeracy matters. Med Decis Making 21: 382-90, 2001.

21. Cuervo LG, Clarke M: Balancing benefits and harms in health care [Editorial]. BMJ 327: 65-6, 2003.

22. Wennberg JE, Fisher ES, Skinner JS: Geography and the debate over Medicare reform. Health Aff (Millwood). 2002; Suppl Web Exclusives W96-114. Acesso em: http://content.healthaffairs. org/cgi/reprint/hlthaff.w2.96vl

23. Covinsky KE, Fuller JD, Yaffe $\mathrm{K}$ et al: Communication and decision-making in seriously ill patients: findings of the SUPPORT project. The Study to Understand Prognoses and Preferences for Outcomes and Risk of Treatment. J Am Geriatr Soc 48: S187-93, 2000.

24. Griffiths F, Green E, Tsouroufli M: The nature of medical evidence and its inherent uncertainty for the clinical consultation: qualitative study. BMJ 330: 511-18, 2005.

25. O'Connor AM, Légaré F, Stacey D: Risk communication in practice: the contribution of decision aids. BMJ 327: 736-47, 2003.

26. Llewellyn-Thomas HA: Patients' health-care decision making: a framework for descriptive and experimental investigations. Med Decis Makig 15: 101-6, 1995.

27. Dowie J: The role of patients' meta-preferences in the design and evaluation of decision support systems. Health Expectations 5: 16-27, 2002.

28. Entwistle V, Sowden A, Watt I: Evaluating interventions to promote patient involvement in decision-making: by what criteria should effectiveness be judged? J Health Serv Res Policy. 3: 100-7, 1998.

29. Whelan T, Gafni A, Charles C, Levine M: Lessons learned from the decision board: a unique and evolving decision aid. Health Expectations 3: 69-76, 2000.

30. Charles C, Gafni A, Whelan T. O'Brien MA: Treatment decision aids: conceptual issues and future directions. Health Expectations 8: 114-125, 2005 .

31. Gafni A, Charles CA, Whelan T: The physician-patient encounter: the physician as a perfect agent for the patient versus the informed treatment decision making model. Soc Sci Med 47: 347-54, 1998 .

32. Whitney SN: A new model of medical decisions: exploring the limits of shared decision making. Med Decis Making 23: 27580, 2003.

33. O'Connor AM, Stacey D, Entwistle V et al: Decision aids for people facing health treatment or screening decisions. Cochrane Database Syst Rev: CD001431 [PMID: 12804407], 2003.

34. Estabrooks C, Thiel E, Pinfold S, Goel V, Sawka C, Williams $\mathrm{J}$ : Consumer decision aids: where do we stand? A systematic review of structured consumer decision aids. Technical Report, Institute for Clinical Evaluative Sciences 1999; (00-01-TR). Toronto. Ontario.

35. Whelan T, Levine M, Gafni A et al: Breast irradiation postlumpectomy: development and evaluation of a decision instrument. J Clin Oncol 13: 847-53, 1995.

36. Edwards A, Elwyn G: How should effectiveness of risk communication to aid patient's decision be judged? A review of the literature. Med Decis Making 19: 428-34 1999.

37. O'Connor A, Drake E, Fiset V, Page J, Curtin D, LlewellynThomas H: Annotated bibliography of studies evaluating 
decision support intervention for patients. Can J Nur Res 29: 113-20, 1997.

38. Gafni A, Birch S: Inclusion of drugs in provincial drug benefit programs: should "reasonable decisions" lead to uncontrolled growth in expenditures? CMAJ 168: 549-51, 2003.

39. Whelan T, Levine M, Willan A et al: Effect of a decision aid on knowledge and treatment decision making for breast cancer surgery. A randomized trial. JAMA 292: 435-41, 2004.

40. Kawamoto K, Houlihan CA, Balas EA, Lobach DF: Improving clinical practice using clinical decision support systems: a systematic review of trials to identify features critical to success. BMJ 330: 765-8, 2005.
41. Molenaar S, Sprangers MA, Postma-Schuit FC, et al: Feasibility and effects of decision aids. Med Decis Making 20: 112-27, 2000.

42. Eckman MH: Patient-centered decision making: a view of the past and a look toward the future. Med Decis Making 21: 2417,2001.

43. Dwight-Johnson M, Lagomasino IT, Aisenberg E, Hay J: Using conjoint analysis to assess depression treatment preferences among low-income latinos. Psychiatr Serv 55: 934-6, 2004.

44. Mayeux EJ, Murphy PW, Arnold C: Improving patient education for patients with low literacy. Am Fam Physician 53: 205-11, 1996. 